\title{
Shape error analysis on the three-dimensional surface part in continuous roll forming
}

\author{
Li-Rong Sun ${ }^{\text {a }}$, Xue-Ping Yan*, Qing-Min Chen \\ Roll Forging Research Institute, Jilin University, Changchun 130025, China \\ a.18843011405@163.com \\ *Corresponding author: 2589018009@qq.com
}

\begin{abstract}
Continuous roll forming (CRF) is a new type of flexible forming technology, which can produce threedimensional surface parts with different curvatures by adjusting a pair of bendable rolls and the distribution of roll gap. In this paper, shape error analysis on the three-dimensional surface part is studied, and the comparison of transverse and longitudinal curvature radii of convex surfaces is expressed. The results show shapes of convex curved surface parts are uniform, while larger curvature is obtained on both sides. Furthermore, the shape error of three-dimensional surface part decreases from the center to the edges, and the radius of formed part is closed to the target radius in the middle area. In addition, the experiments of convex curved surface parts are carried out, and the experimental results show the validity of the simulation results.
\end{abstract}

Keywords-shape error; continuous roll forming; threedimensional surface; radius of curvature

\section{INTRODUCTION}

Continuous roll forming is a new forming process for threedimensional sheet metal, combining of multi-point adjustment and rolling technology $[1,2]$. The sheet metal is compressed between the upper and lower bendable rolls, and the transverse bending deformation is generated by the configuration of the flexible rolls, while the longitudinal deformation results form the non-uniform longitudinal strain caused by the non-uniform distribution of the roll gap [3]. Three-dimensional surface parts with different curvatures can be formed in a set of continuous roll forming equipment, which can satisfy the demand of product variety and small batch with high efficiency and low cost $[4,5]$.

The basic principle and theory of CRF has been derived by Cai et al. in the previous work [6,7], and the relationship of the roll gap distribution and curvature of sheet metal part has been established. Since the transverse and longitudinal curvatures of sheet metal parts are determined by the distribution of the roll gap, the reasonable design of the roll gap is the key to forming qualified parts [8]. In the rolling process, the sheet metal can be fed by friction between blank and a pair of rolls, then shaped into three-dimensional surface part with the rotation of the upper and lower flexible roll, bent in horizontal and vertical directions simultaneously [9]

\section{PRINCIPLE OF CONTINUOUS ROLL FORMING}

The distribution of roll gap adjusted by multiple discrete controlled points is determined by the transverse and longitudinal curvature of the target part, and schematic of continuous roll forming device is shown in Fig.1a. When the roll gap is relatively smaller in the middle and larger on both edges, convex curved surface parts are produced, on the contrary, saddle surface parts are obtained [7]. The distribution of roll gap is shown in Fig.1b.

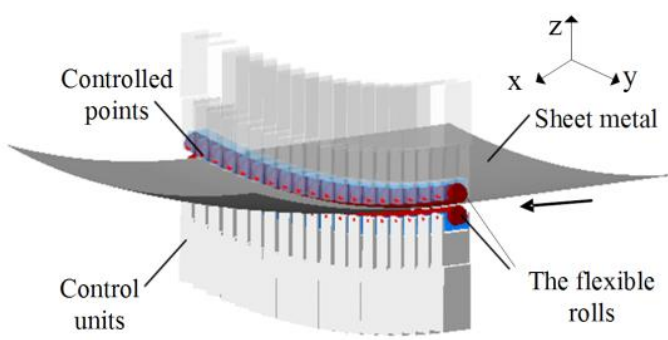

(a) Schematic of continuous roll forming device

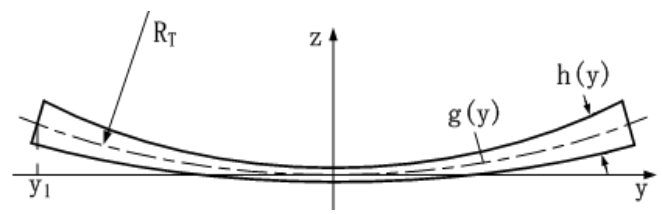

(b) Cross-section shape of roll gap

Fig.1 Principle of continuous roll forming

The transverse and longitudinal curvature radii of the target parts are $R_{T}$ and $R_{L}$ respectively. The function of neutral axis is denoted as $\mathrm{g}(\mathrm{y})$ (as shown in (1)), and the function of roll gap distribution is expressed as $\mathrm{h}(\mathrm{y})$ (as shown in (3) and (4)), where the $y$-axis is in the direction perpendicular to the rolling direction and $\mathrm{z}$-axis is the thickness direction of the parts [10].

$$
g(y)=R_{T}\left[1-\sqrt{1-\left(y / R_{T}\right)^{2}}\right]
$$

The compression ratio is defined as $\varepsilon(y)$,

$$
\varepsilon(y)=(H-h) / H=\Delta h(y) / H
$$

where $\mathrm{H}$ is the original thickness of formed part. The $\varepsilon_{\text {min }}$ $\left(\varepsilon_{\min }=0\right.$ ) on the edges of convex curved surface parts is the minimum compression ratio, and $\varepsilon_{\min }\left(\varepsilon_{\min }=0\right)$ in the center of saddle surface parts.

Therefore, the functions of roll gap width distribution of convex curved surface parts and saddle surface parts are expressed as 


$$
\begin{aligned}
& h(y)=\left\{1-R_{T} / R_{L}\left[\sqrt{1-\left(\mathrm{y} / \mathrm{R}_{T}\right)^{2}}-\sqrt{1-\left(y_{1} / \mathrm{R}_{T}\right)^{2}}\right]\right\} H \\
& h(y)=\left\{1-R_{T} / R_{L}\left[1-\sqrt{1-\left(y / R_{T}\right)^{2}}\right]\right\} H
\end{aligned}
$$

\section{NUMERICAL SIMULATIONS OF CONVEX CURVED SURFACE PARTS}

Numerical simulations of CRF were performed under ABAQUS/Explicit environment, and 1/2 finite element model was adopted. The dimension of the sheet metal shaped into convex curved surface part was $200 \mathrm{~mm} \times 130 \mathrm{~mm}$ with the thickness $2 \mathrm{~mm}$, and the material was mild steel 08AL. The coordinates of the section curves were extracted, and the shape errors of three-dimensional surface part were calculated to analyze the results of numerical simulation. Meanwhile, longitudinal and transverse curvature radii were compared with the target radii of the desired part.

\section{A. Shape error analysis on convex curved surface part in $C R F$}

In the process of finite element numerical simulation, longitudinal target radius of convex curved surface part is $350 \mathrm{~mm}$, and transverse target radius is $300 \mathrm{~mm}$. Fig. 2 shows convex curved surface part of numerical simulation and Fig.3 shows the extracting contour in different positions of longitudinal and transverse directions. Due to adopting 1/2 finite element model, in the longitudinal direction, extract five evenly distributed lines $\left(Z_{0}-Z_{4}\right)$ located the same side of symmetry axis $\left(Z_{0}\right)$. Extract eleven evenly distributed lines from the entry end to the exit end, in which $\mathrm{M}_{0}$ is in the middle.

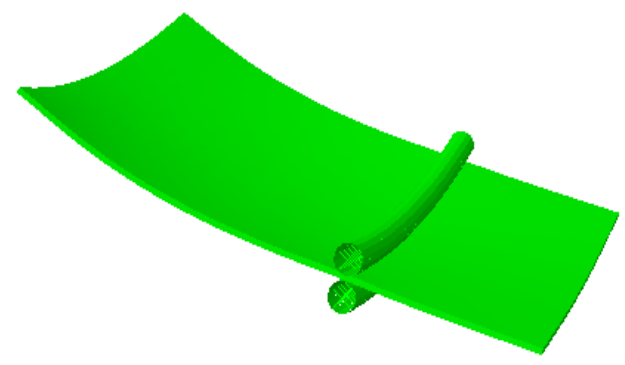

(a) Formed part in rolling

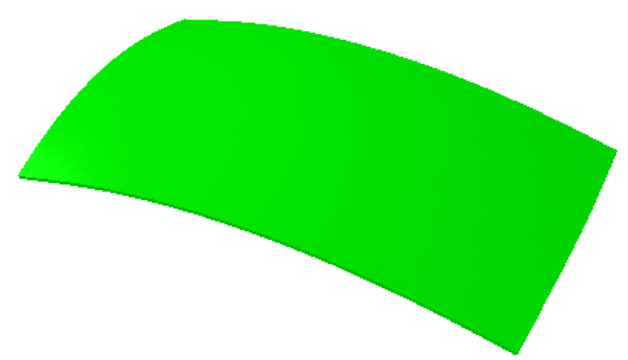

(b) The convex curved surface part of numerical simulation

Fig.2 The convex curved surface part in continuous roll forming

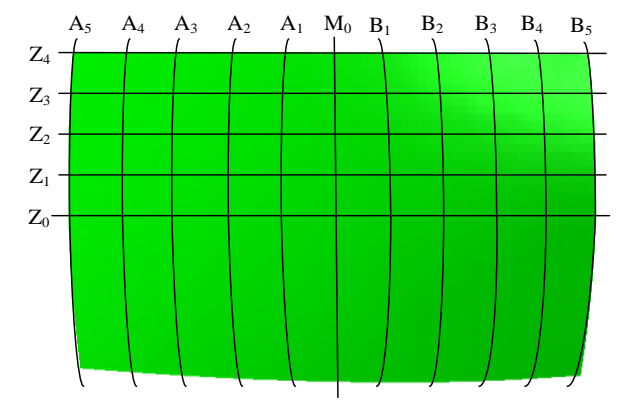

Fig.3 Positions of section lines of the convex curved surface part

The coordinates of each point of the section curves in Fig.3 are extracted. In order to express intuitively the shapes of the section lines, translate the starting points of the curves to the same start point, and the comparison result of different section lines in longitudinal direction is shown in Fig.4a. In general, the shapes of longitudinal section lines are uniform, while larger curvature is obtained on both sides in local amplification figure. In Fig. $4 b$, translate the vertexes of the curves to the same point, the transverse curvature decreases gradually from the entry end to the exit end.

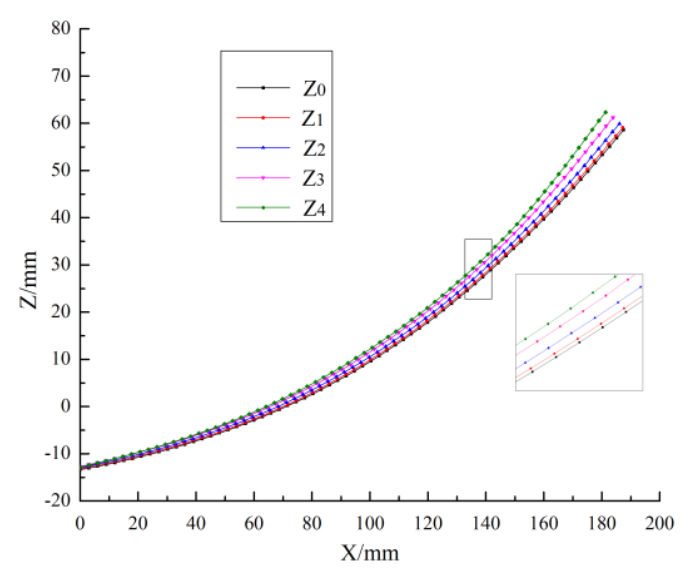

(a) The shape of longitudinal curves

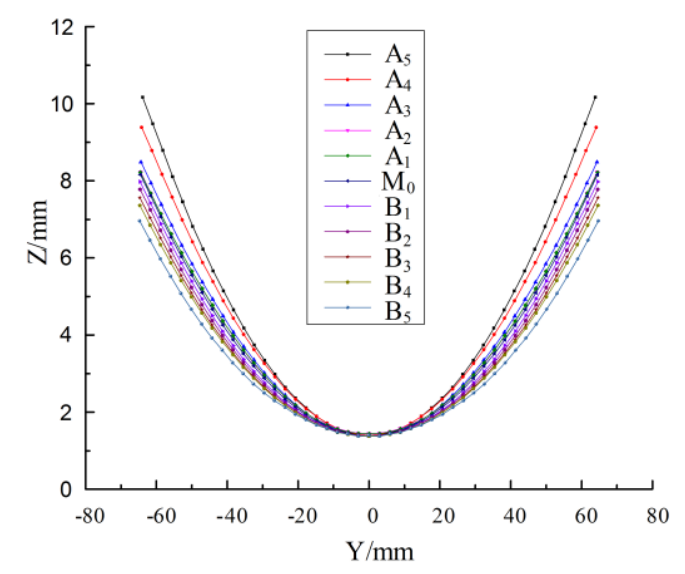

(b) The shape of transverse curves

Fig.4 Schematic illustration of formed part shape in different positions: 
Fig.5 shows shape error distribution along section lines, and the relative error on the edges is larger than the middle, while the total error is relatively small less than $0.6 \mathrm{~mm}$. In the transverse direction, shape error is minimal in the middle area, and the total error is less than $0.8 \mathrm{~mm}$. Therefore, convex curved surface part in numerical simulation embraces high precision.

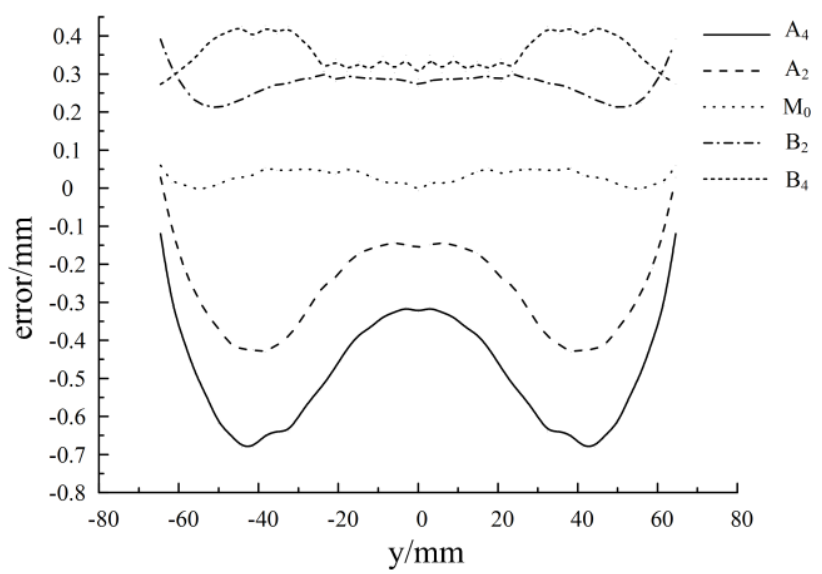

(a) Shape error of longitudinal section lines

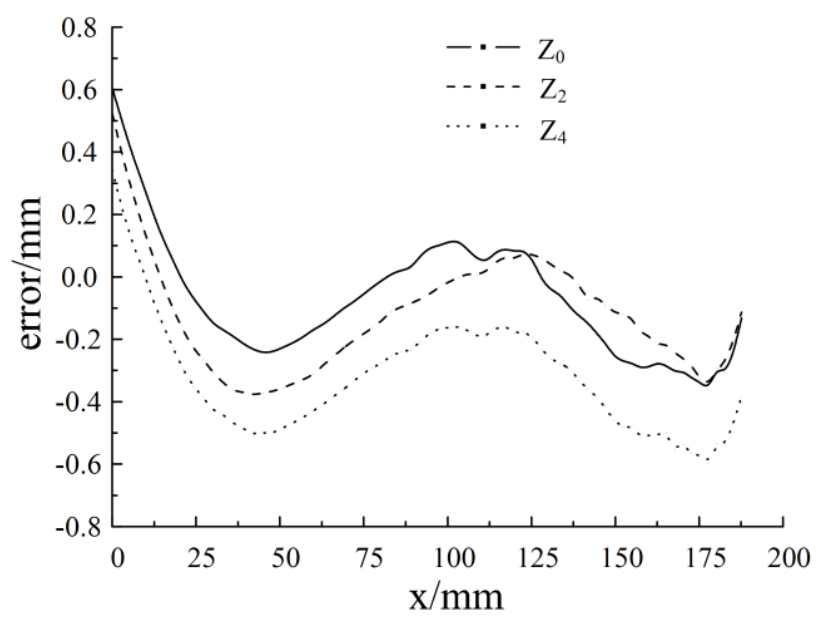

(b) Shape error of transverse section lines

Fig.5 Shape error distribution of section lines

\section{B. Comparison of curvature radii and target radii}

Longitudinal target radius of convex curved surface part is $350 \mathrm{~mm}$, and transverse target radius is $300 \mathrm{~mm}$ in numerical simulation. By fitting the curvature radii of the extracting contour and comparing it with target curvature radii, analyze the shape errors in longitudinal and transverse directions. Longitudinal and transverse curvature radii of different positions are shown respectively in Table 1 and Table 2.

Table 1 Longitudinal curvature radius of different positions

\begin{tabular}{cccccc}
\hline position & $\mathrm{Z}_{0}$ & $\mathrm{Z}_{1}$ & $\mathrm{Z}_{2}$ & $\mathrm{Z}_{3}$ & $\mathrm{Z}_{4}$ \\
\hline $\mathbf{R}(\mathbf{m m})$ & 352 & 349 & 343 & 332 & 317 \\
\hline
\end{tabular}

Table 2 Transverse curvature radius of different positions

\begin{tabular}{lcccccc}
\hline position & $\mathrm{A}_{5}$ & $\mathrm{~A}_{4}$ & $\mathrm{~A}_{3}$ & $\mathrm{~A}_{2}$ & $\mathrm{~A}_{1}$ & $\mathrm{M}_{0}$ \\
\hline $\mathbf{R}(\mathbf{m m})$ & 244 & 258 & 291 & 305 & 306 & 308 \\
\hline position & $\mathrm{B}_{1}$ & $\mathrm{~B}_{2}$ & $\mathrm{~B}_{3}$ & $\mathrm{~B}_{4}$ & $\mathrm{~B}_{5}$ & \\
\hline $\mathbf{R}(\mathbf{m m})$ & 316 & 327 & 338 & 350 & 369 & \\
\hline
\end{tabular}

Table 1 shows the longitudinal curvature radii decrease gradually from the middle to two sides, and the longitudinal curvature radii in intermediate region is closer to the target radius $\left(R_{L}=350 \mathrm{~mm}\right)$, so the shape of simulated part is better in central region than marginal region. Table 2 shows the transverse curvature radii increase gradually from the entry end to the exit end, and the curvature radii of middle area are approximate to the target radius $\left(\mathrm{R}_{\mathrm{T}}=300 \mathrm{~mm}\right)$ of the desired part. In general, the longitudinal and transverse curvature radii are closed to the target radius of the desired part in the middle area, and the shape of simulated part is better in central region.

\section{EXPERIMENTAL INVESTIGATIONS}

Numerical simulation results indicate the shape of convex curved surface part is uniform, and the shape error is relatively less in the middle area. Meanwhile, the radius of formed part is closed to the target radius of the desired part in the middle area, and the longitudinal curvature radii decrease from the center to the both sides and the transverse curvature radii increase gradually from the entry end to the exit end. In experiment, the sheet metal with the dimension of $400 \mathrm{~mm} \times 330 \mathrm{~mm}$ and the thickness $1.8 \mathrm{~mm}$ was formed the convex curved surface part with longitudinal target radius $500 \mathrm{~mm}$ and transverse target radius $3500 \mathrm{~mm}$. Experimental formed part is shown in Fig.6a, which is high forming quality without wrinkling.

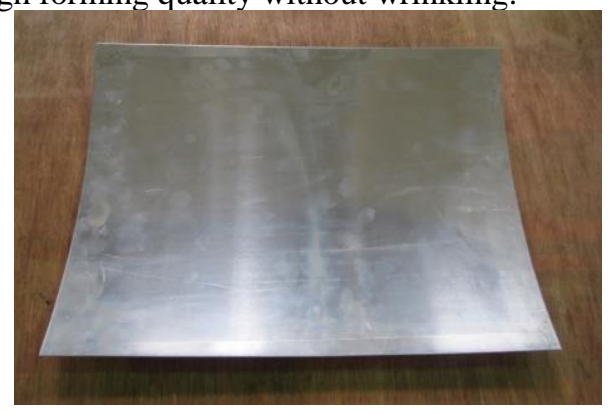

(a) Experimental formed part

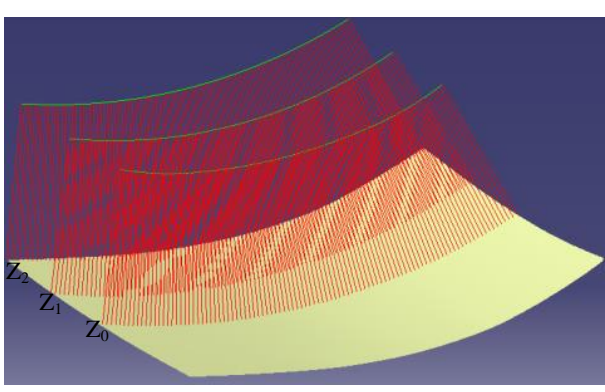

(b) Measurement of curvature radius of shaped part

Fig. 6 the experiments of convex curved surface parts 
Using PRO CMM system to scan the surface of formed part, point cloud data of convex surface part was obtained. The point cloud data were triangulated in CATIA, then produce threedimension surface by reverse engineering. Different section curves were analyzed the radii in the longitudinal and transverse directions, as shown in Fig $6 \mathrm{~b}$.

Table 3 Longitudinal curvature radius of different positions

\begin{tabular}{cccc}
\hline positions & $\mathrm{Z}_{0}$ & $\mathrm{Z}_{1}$ & $\mathrm{Z}_{2}$ \\
\hline $\mathbf{R}(\mathbf{m m})$ & 510 & 478 & 457 \\
\hline
\end{tabular}

Table 4 Transverse curvature radius of different positions

\begin{tabular}{cccccc}
\hline positions & $\mathrm{A}_{2}$ & $\mathrm{~A}_{1}$ & $\mathrm{M}_{0}$ & $\mathrm{~B}_{1}$ & $\mathrm{~B}_{2}$ \\
\hline $\mathbf{R}(\mathbf{m m})$ & 3356 & 3568 & 3747 & 3785 & 3803 \\
\hline
\end{tabular}

Radii in different section lines are expressed in Table 3 and Table 4 by shape analysis. In longitudinal direction, radii reduce from $510 \mathrm{~mm}$ in the middle to $457 \mathrm{~mm}$ in the edges. Accordingly, in transverse direction, radii increase from $3356 \mathrm{~mm}$ to $3803 \mathrm{~mm}$ from the entry end to the exit end. The radii in the middle region are closer to the target radii in both directions, which are in good agreement with those of simulation.

\section{CONCLUSIONS}

By analyzing the results of numerical simulations and experiments of the convex curved surface parts, the following conclusions are acquired:

(1) The shapes of convex curved surface parts are uniform, while larger curvature is obtained on both sides. Furthermore, the shape error is relatively small, and convex curved surface parts possess high quality in middle area.

(2) The radii are closed to the target radii in intermediate region, and the radii of longitudinal direction decrease gradually from the middle to the both edges, and the radii of transverse direction increase from the entry end to the exit end.

(3) The experimental results show the validity of the simulation results.

\section{REFERENCES}

[1] S.J. Yoon, D.Y. Yang, Development of a highly flexible incrementalroll forming process for the manufacture of a doubly curvedsheet metal, Cirp Ann-Manuf Technol. 52 (2003) 201-204.

[2] D.S. Shim, D.Y. Yang, K.H. Kim, S.W. Chung, M.S. Han, Investigation into forming sequences for the incremental formingof doubly curved plates using the line array roll set (LARS) process, Int J Mach Tool Manuf. 50 (2010) 214-218.

[3] Z.Y. Cai, M.Z. Li, Y.W. Lan. Three-dimensional sheet metal continuous forming process based on flexible roll bending: Principle and experiments. Journal of materials processing technology. 212 (2012) 120-127

[4] Z.Y. Cai, D.B. Guan, M. Wang, et al. A novel continuous roll forming process of sheet metal based on bended rolls. International Journal of Advanced Manufacturing Technology. 73 (2014) 1807-1814

[5] Z. Sui, Z.Y. Cai, Y.W. Lan, et al. Simulation and software design of continuous flexible roll bending process for three dimensional surface parts. Materials\&Design. 54 (2014) 498-506

[6] Z.Y. Cai, M. Wang, M.Z. Li, Study on the continuous roll forming process of swept surface sheet metal part, Journal of Materials Processing Technology. 214 (2014) 1820-1827.

[7] Z.Y. Cai, M.Z. Li, Principle and theoretical analysis of continuous roll forming for three-dimensional surface parts, Science ChinaTechnological Sciences. 56 (2013) 351-358.

[8] M. Wang, Z.Y. Cai, L.L. Li, M.Z. Li, Longitudinal bending deformation analysis of sheet metal in continuous roll forming process, International Journal of Advanced Manufacturing Technology. 80 (2015) 467-476.

[9] D.M. Wang, M.Z. Li, Z.Y. Cai. Continuous-forming method for threedimensional surface parts combining rolling process with multipointforming technology. International Journal of Advanced Manufacturing Technology. 72 (2014) 201-207

[10] Z.Y. Cai, Z Sui, F.X. Cai, Continuous flexible roll forming for threedimensional surface part and the forming process control, International Journal of Advanced Manufacturing Technology. 66 (2013) 393-400. 